\title{
THE QUALITY OF BEHAVIORAL AND ENVIRONMENTAL INDICATORS USED TO INFER THE INTENTION TO CHANGE LANES
}

\author{
Matthias J. Henning, ${ }^{1}$ Olivier Georgeon, ${ }^{2}$ \& Josef F. Krems ${ }^{1}$ \\ ${ }^{1}$ Chemnitz University of Technology \\ Chemnitz, Germany \\ ${ }^{2}$ INRETS (Institut National de Recherche sur les Transports et leur Sécurité) \\ Bron, France \\ E-mail: matthias.henning@phil.tu-chemnitz.de
}

\begin{abstract}
Summary: This study focuses on the examination and comparison of selected behavioural and environmental indicators that predict the intention to change lanes. These indicators were chosen from previous driving studies and driver models. The data were gathered in a field study with an instrumented car that can log data from the driver, the car, and the environment. The collected data were analysed and modelled with the help of a "knowledge discovery framework" (Georgeon, Mille, \& Bellet, 2006). The first analysis of all lane changes caused by a slow leading vehicle focuses on the following indicators: glance to the left outside mirror, turn signal, and lane crossing. It is shown that the glance to the left outside mirror could serve as a predictor with a high potential to get information about the intention to change lanes in a very early stage. However, it is important to combine this predictor with additional predictors to avoid a high false alarm rate.
\end{abstract}

\section{INTRODUCTION}

The increase in the use of Advanced Driver Assistance Systems (ADAS) and In-Vehicle Information Systems (IVIS) in recent years has created the need for information about the driver and the traffic situation. To augment the benefit to traffic safety and the driver's acceptance of such systems, these ADAS and IVIS should react according to the traffic situation and according to the driver's intention. For example, a Lane Departure Warning System (LDW) warns the driver if he/she leaves the current lane. However, if the driver wants to execute a lane change, the system should not warn the driver.

A lane change is a ubiquitous driving manoeuvre in common driving environments. It combines many critical features of driving such as low-level control, monitoring, and decision making. Lane change is defined by a more or less stable sequence of actions, which begins with a motivation to change lanes (for instance a slow leading vehicle), followed by a gathering of information about the surrounding traffic situation and the decision whether to change lanes or not (e.g., McKnight \& Adams, 1970).

Several sources of data could be used to infer a driver's intention to change lanes. First there are car data; for example, using the turn signal could be a valid predictor of the intention to change lanes. However, drivers do not use the turn signal in every lane-change situation. In a field study, Olsen (2003) reported that drivers tend to indicate a lane change in only $65 \%$ of all cases. Other predictors could be steering wheel angle or acceleration (Liu \& Pentland, 1997). A second source 
of data is the car's external environment (Baker \& Carter, 1997). The surrounding traffic, the lane position, or geographical position can have some indicative power about the intention to change lanes. Third, drivers themselves could serve as a source. In particular, eye movement behaviour could be a rich source of information about the current goals and motives of the driver (Henning, Schweigert, Baumann, \& Krems, 2006).

There have been several attempts to build algorithms to predict a lane change manoeuvre. In a simulator study, Liu and Pentland (1997) developed Hidden Markov Models (HMMs) to predict a lane change to the left out of several other driving manoeuvres. Prediction accuracy was 50\% $0.5 \mathrm{sec}$ after the onset of the manoeuvre, but increased in accuracy over time. Liu and Pentland only used information about the steering wheel for their models. Oliver and Pentland (2000) also used HMMs to predict a lane change manoeuvre to the left among other manoeuvres in real traffic situations. Here, prediction accuracy was $23.5 \% 0.1 \mathrm{sec}$ before the manoeuvre took place. These authors used car data (speed, acceleration, brake, gear, and steering angle) and gaze information. McCall, Wipf, Trivedi, and Rao (2004) used Sparse Bayesian Learning to develop an algorithm to detect the intention to change lanes to the left. Information used in this model was gas pedal position, brake pedal depression, longitudinal acceleration, vehicle speed, steering angle, yaw rate, lateral acceleration, road curvature metric, heading, lateral lane position up to $20 \mathrm{~m}$ ahead, and side-to-side and up-down head movement. Real traffic data of only three participants were used, and an accuracy of approximately $95 \%$ was achieved, with 5\% false alarms. Salvucci (2004) proposed a "mind-tracking architecture" based on a cognitive model of driver behaviour implemented in the ACT-R cognitive architecture (Anderson \& Lebiere, 1998). He reported a hit rate of $85 \%$ with $4 \%$ false alarms by using information from the car, the environment, and the driver in a driving simulator.

All these attempts to build valid algorithms are dissatisfactory, as those studies suffer from a very small sample size or were done in driving simulators. Studies that were done in real traffic with a sufficient number of participants had low hit rates.

The goal of the present study is to focus on the impact of specific indicators and their position in the lane-change sequence. The present article describes in more detail the timeline of the following indicators: slow leading vehicle, glance to the left outside mirror, turn signal, and lane crossing. Those behavioural indicators were chosen according to the Intent Detection Framework proposed by Smith and Zhang (2004).

\section{METHOD}

Participants. Data of 22 participants ages 24 to 58 years were recorded (MEAN $=33.8$ years, $\mathrm{SD}=10.1$ years). Ten were female, and 12 were male. Their driving experience ranged between 2 and 39 years $(\mathrm{MEAN}=13.4$ years, $\mathrm{SD}=9.7$ years $)$, with an annual quantum of driving from 2,000 to $50,000 \mathrm{~km}(\mathrm{MEAN}=13,136 \mathrm{~km}, \mathrm{SD}=10,508 \mathrm{~km})$.

Instrumented car. A Renault Scénic was equipped to record and synchronize sensor data and videos. The synchronization was acheived by using the time code from the video frame. The logged sensor data came from car dynamics (speed, acceleration, deceleration, yaw rate, and inclination), driver's behaviour (eye movement, steering wheel position, pedal use, and turn 
signal), and environmental data (distance to car ahead, GPS positioning). Video signals were recorded from five sources: stereo-vision camera with radar for distance estimation to obstacles (top left), the front view (top right), the rear view (lower left - upper part), the view from the left outside mirror down to the surface of the road (lower left - lower part), and the view of the participant's head with indications of the eye-tracker (lower right). Figure 1 shows the video logged during the experiment.

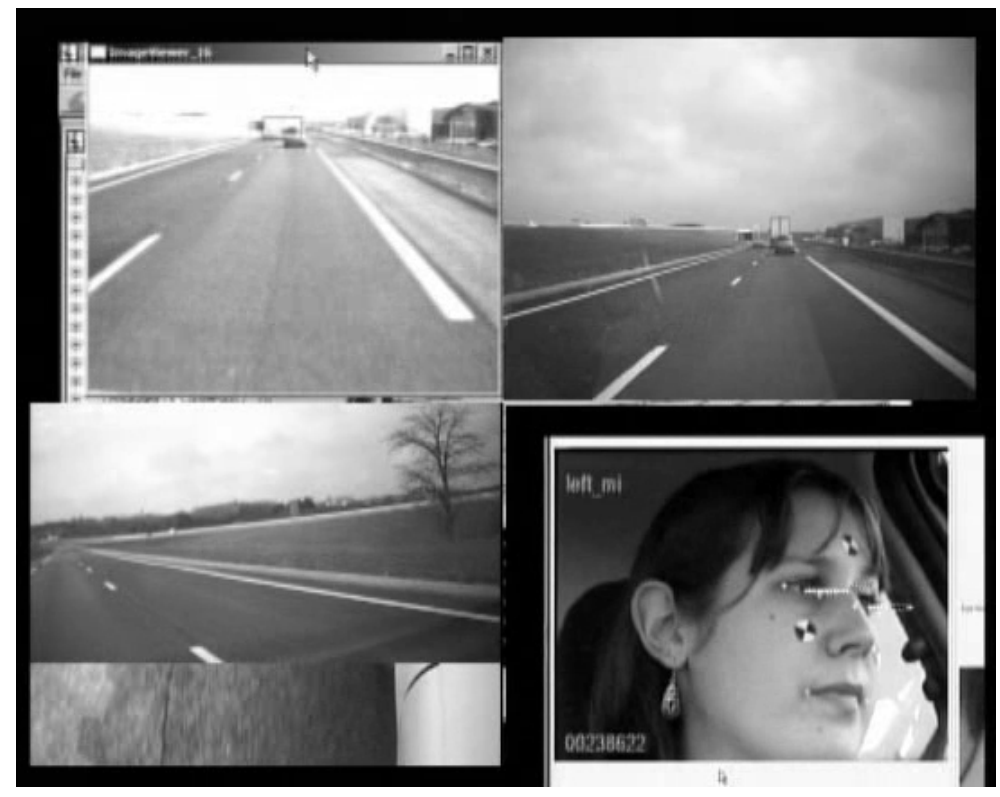

Figure 1. Video sources from: stereo-vision camera with radar for distance estimation to obstacles (top left), the front view (top right), the rear view (lower left - upper part), the view from the left outside mirror down to the surface of the road (lower left - lower part), and the view of the participant's head with the glance direction of the eye (lower right)

Test course. The field study was conducted in the area of central France around the city of Lyon. The subjects drove on a multi-lane motorway between Bron and the Lyon International Airport Saint Exupéry in both directions. The total length of this course is about $50 \mathrm{~km}$. The speed limit varies between $90 \mathrm{~km} / \mathrm{h}$ and $130 \mathrm{~km} / \mathrm{h}$.

Procedure. Participants were first informed about the goal of the study. They were told to drive like they normally do. Participants then received information about the car. The experimenter calibrated the eye tracker and started the data logging. During test driving, an experimenter in the back of the car watched the driving scene and indicated the motive for a lane change by pressing a button. (e.g., a slow leading vehicle). This signal was logged with a time stamp (not seen by the driver) and was used for the post-experiment interview. After the test drive, the data were immediately processed in order to find the sequences with a motive to change lanes in the video. With this information participants were questioned in one of two ways, depending on if there was a lane change following or not. If there had been no lane change, the participants were asked whether they had thought about executing a lane change or not. In the case of thinking about executing a lane change, they were asked to show the starting point and the point at which they had decided not to change lanes in the video. If there had been a lane change, participants were asked to show the point where they had started to think about a lane change and the point after 
the execution of a lane change where they had stopped thinking about the lane change. After the interview, participants filled out a questionnaire on demographic data. Finally they were debriefed and thanked.

Data Analysis. The analysis was performed using a "knowledge discovery framework" (Georgeon, Mille, \& Bellet, 2006), which enables the user in a first step to visualize the indicators, chosen a priori, and the driving video at the same time. In a second step, it allows the researcher to apply rules to test certain combinations of indicators. For this reason, a softwaretool called ABSTRACT (Analysis of Behavior and Situation for menTal Representation Assessment and Cognitive acTivity modeling) was developed by INRETS and the Chemnitz University of Technology (see Figure 2).

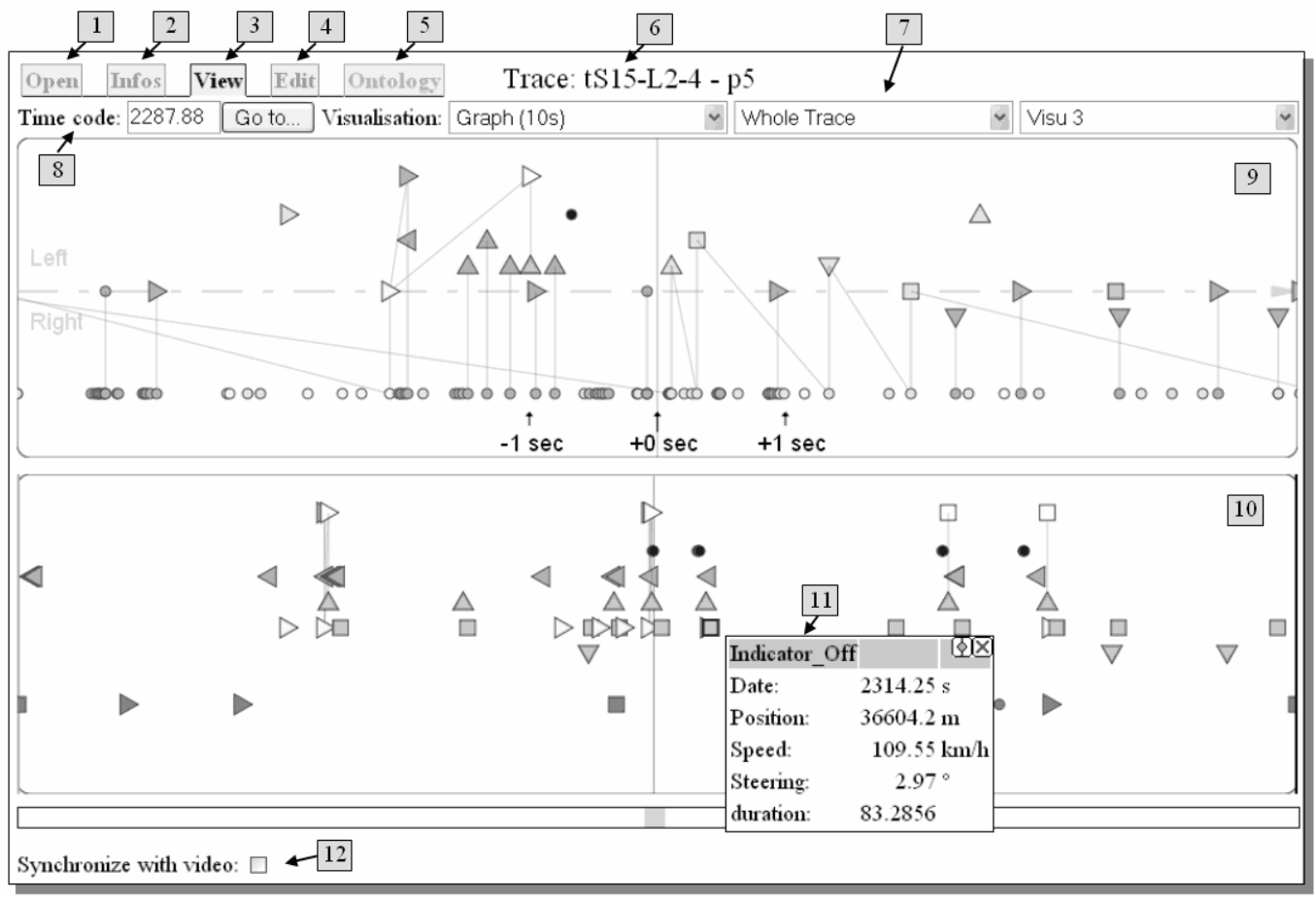

Figure 2. Screenshot of ABSTRACT: (1) select a trace (6), (2) view global information about it, (3) view its content under different formats, (4) transform it by defining and applying rules of transformation, (5) define desired symbols in an ontology, (7) choose different visualisation modules, (8) go to a specific time code, the visualisation module (10) shows the whole trace with the higher level symbols, the (9) shows a zoom into an interval of 10 seconds, a click on the symbols shows the properties (11), synchronize with the video shown in Figure 1 (12)

With this software, the user is able to perform a step by step process of abstraction starting from the raw data. In the visualisation module (9), the circles at the bottom are events of the basic level of abstraction (e.g., minima and maxima in the sensor data), and the triangles and squares above are the symbols of the higher level, which are inferred from the basic level. Detailed descriptions of those symbols are shown in the qualitative description of a lane-change manoeuvre. 


\section{RESULTS}

\section{Quantitative description of a lane change manoeuvre}

Mental representation of a lane change. Altogether 194 lane changes to the left were analyzed. The durations between the starting point of the lane-change schema ('start thinking about a lane change') and the actual lane crossing for all lane changes are shown in Figure 3.

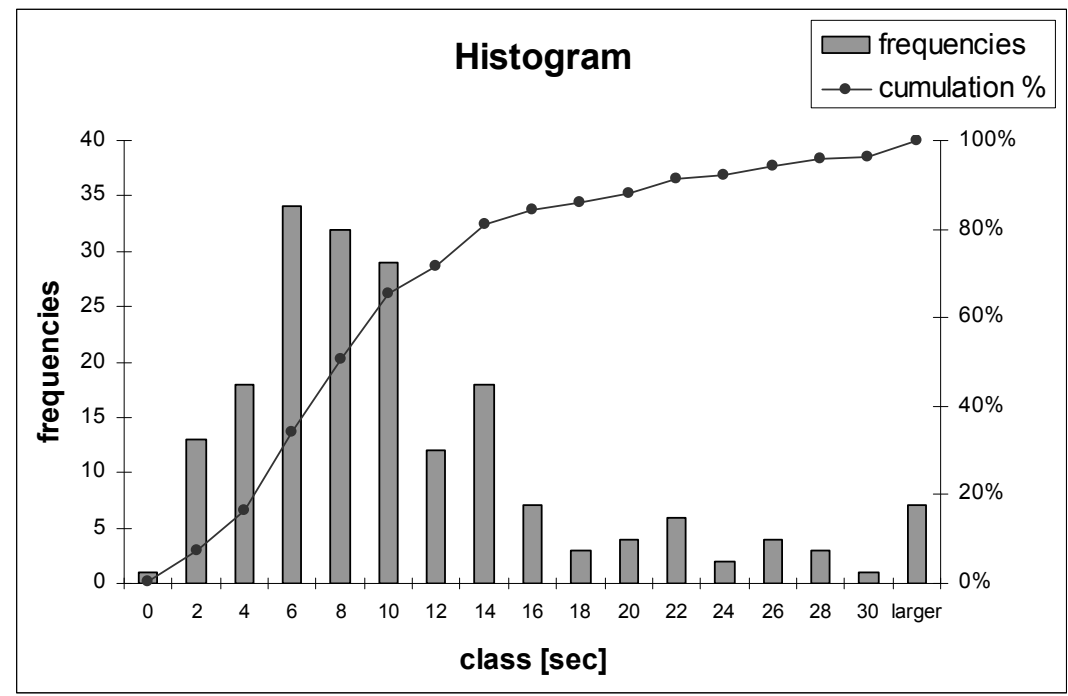

Figure 3. Histogram of the duration of all lane-change schemas

The mean value is $10.53 \mathrm{sec}(\mathrm{SD}=10.18 \mathrm{sec})$ with a median of $7.82 \mathrm{sec}$. The minimum was at $0 \mathrm{sec}$ and the maximum at $88.64 \mathrm{sec}$.

Timeline of a lane-change manoeuvre. All lane-change manoeuvres were caused by a slow leading vehicle and were performed to pass this vehicle. This study focuses on the following indicators: a) first glance to the left outside mirror, b) turn signal, and c) the actual lane crossing. There was at least one glance to the left outside mirror in $99.0 \%$ of all lane changes, and the turn signal was used in $99.0 \%$. In $87.1 \%$ of all lane changes, the glance to the left outside mirror preceded the turn signal; in $11.3 \%$ the order was preserved. Three lane changes were not preceded by a glance to the left outside mirror or a signal or both. The mean duration between the first glance to the left outside mirror and the lane crossing was $6.12 \mathrm{sec}$ $($ MEDIAN $=4.00 \mathrm{sec}, \mathrm{SD}=7.01 \mathrm{sec})$. The mean duration between the onset of the left turn signal and the lane crossing was $2.28 \mathrm{sec}(\mathrm{MEDIAN}=2.00 \mathrm{sec}, \mathrm{SD}=2.17 \mathrm{sec}$ ).

\section{Qualitative description of a lane-change manoeuvre}

As an example, two lane-change sequences were modelled with the help of ABSTRACT. The first schema (Figure 4) is characterized by the fact that it begins in a situation where the driver had to drive below his preferred speed. In this schema, the acceleration-associated glance into his left outside mirror appears as a good predictor of the lane change. One second after this possible predictor, the participant switched on the left turn-signal. 


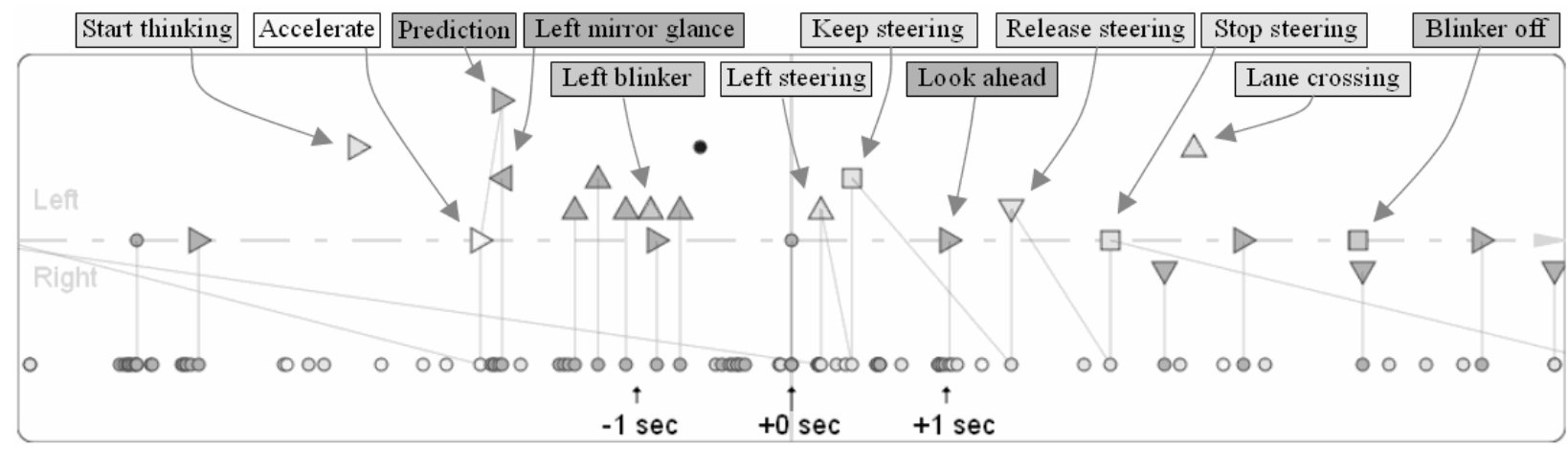

Figure 4. Lane-change sequence with acceleration

In the second schema (Figure 5), the participant is not blocked by the obstacle and he performs the lane change "on the fly." In this case, there is no predictor before the turn signal itself. Nevertheless, the turn signal appears to be a sufficient predictor, since it is switched on in anticipation several seconds before the manoeuvre.

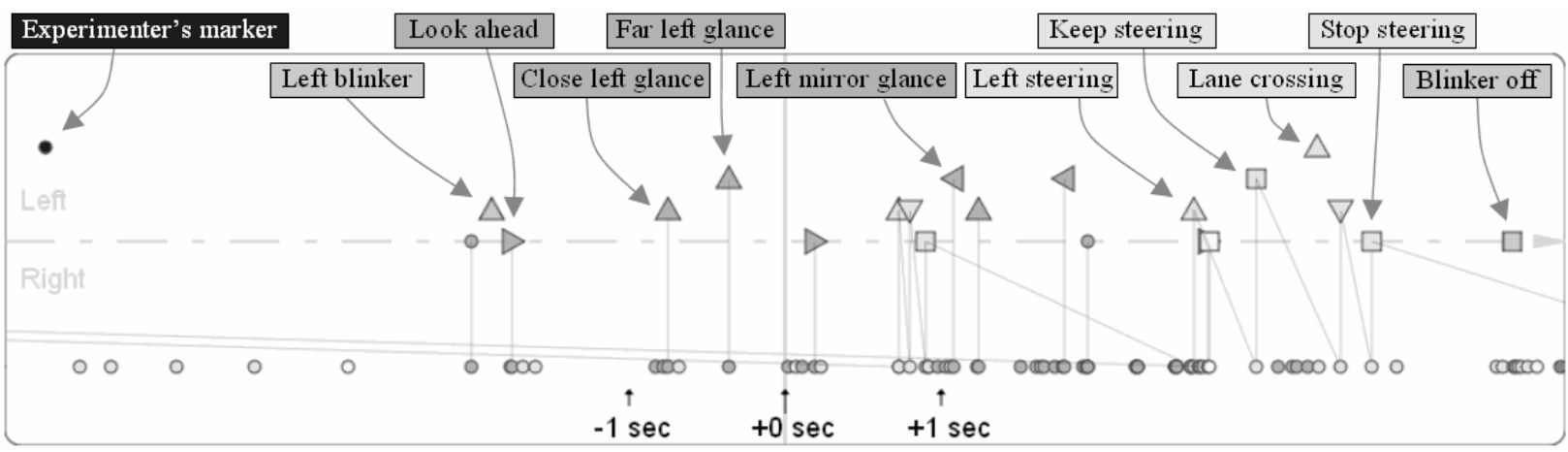

Figure 5. Lane change sequence without any acceleration

\section{CONCLUSIONS}

The preliminary results of the analysis suggest that there is a potential for several types of indicators and combinations of indicators to predict the intention to change lanes. It is shown that the left turn signal and the glance to the left outside mirror are two strong indicators. The high use of the turn signal in this study seems due to the presence of the experimenter. Olsen (2003) reports a much lower frequency in using the turn signal in naturalistic driving conditions. In addition, it is shown that the glance to the left outside mirror is of high potential to increase the predictive power in two ways. On the one hand, the glance to the left outside mirror allows for an earlier prediction than the left turn signal. On the other hand, the glance to the left outside mirror represents a chance to predict the intention to change lanes even if the driver does not use the turn signal. However, this glance to the left outside mirror is also present to a certain extent if there is no intention to change lanes. In two studies Henning et al. (2006) reported a probability for a lane change given a glance to the left outside mirror of $p=0.40$, respectively $p=0.79$. It shows that $60 \%$ respectively $21 \%$ of all glances to the left outside mirror are within the baseline. The predictive power of the glance to the left outside mirror could be increased with the help of 
other predictors like the approaching of slow leading cars and the specific patterns of a driver. A detailed analysis and discussion of this topic can be found in Henning and Krems (in prep.).

\section{ACKNOWLEDGMENTS}

This project was granted by the European Union and part of Network Of Excellence (NOE) HUMANIST (HUMAN centred design for Information Society Technologies applied to road transport).

\section{REFERENCES}

Anderson, J.R., \& Lebiere. C. (1998). The atomic components of thought. Hillsdale, NJ: Erlbaum.

Baker, M., \& Carter. M. (1997). Development of a Lane Changing Algorithm for Evaluating the Impact of ICC. Report, Science Applications International Corporation, McLean, VA, for Volpe National Transportation Systems Center, Cambridge, MA.

Georgeon, O., Mille, A., \& Bellet, B.T. (2006). Analyzing behavioral data for refining cognitive models of operator. Paper presented at the 17th International Conference on Database and Expert Systems Applications (DEXA'06), Krakow, Poland.

Henning, M.J., Schweigert., M., Baumann, M., \& Krems, J.F. (2006). Eye-glance patterns during lane change manoeuvres. Paper presented at the 11th conference on Vision in Vehicles, Dublin, Ireland.

Henning, M.J., \& Krems, J.F. (2007). The impact of behavioural indicators to infer the lane change intention. Paper in preparation for publication.

Liu, A. \& Pentland, A.P. (1997). Towards Real-Time Recognition of Driver Intention. Paper presented at the 1997 IEEE Intelligent Transportation Systems Conference, Boston, MA.

McCall, J., Wipf, D., Trivedi, M., \& Rao, B.D. (2004). Lane Change Intent Analysis Using Robust Operators and Sparse Bayesian Learning (Technical Report). San Diego La Jolla: University of California.

McKnight, A.J., \& Adams, B.B. (1970). Driver Education Task Analysis: Volume I: TaskDescriptions. (Report No. DOT-HS-800-367). Washington, DC: National Highway Traffic Safety Administration.

Oliver, N., \& Pentland, A. (2000). Graphical models for driver behavior recognition in a smartcar. Proceedings of the IEEE lntelligent Vehicles Symposium.

Olsen, E.C.B. (2003). Modeling Slow Lead Vehicle Lane Changing. (Unpublished dissertation.) Virginia Polytechnic Institute and State University, Blacksburg VA.

Salvucci, D.D. (2004). Inferring driver intent: A case study in lane-change detection. Proceedings of the Human Factors Ergonomics Society 48th Annual Meeting.

Smith, M., \& Zhang, H. (2004). SAVE-IT - SAfety VEhicles using adaptive Interface Technology (Task 9): Intent Inference. Washington, DC: National Highway Traffic Safety Administration. 